\title{
La Stratégie nationale de recherche et d'innovation : une opportunité pour Natures Sciences Sociétés?
}

Depuis une vingtaine d'années, le monde de la recherche multiplie les réflexions stratégiques, au sein des différents organismes ou au niveau national (volet recherche de la Stratégie nationale de développement durable [SNDD], Stratégie nationale de recherche sur la biodiversité....). Cependant, jusqu'à présent, chacune de ces réflexions restait cantonnée dans un champ limité à une institution ou à une problématique. Ce n'est plus le cas aujourd'hui, le gouvernement voulant doter le pays d'une stratégie pour l'ensemble du secteur de la recherche, ce que traduit la discussion en cours sur une Stratégie nationale de recherche et d'innovation (SNRI). Même si le texte final n'est pas encore validé à l'heure où nous écrivons ces lignes, de nombreux documents sont déjà disponibles sur le site du ministère de l'Enseignement supérieur et de la Recherche (http://forums.snri.enseignementsup-recherche.gouv.fr/), ce qui nous autorise à faire un premier commentaire de ces textes que peu de chercheurs connaissent.

Comme toute stratégie, la SNRI part d'une analyse des enjeux sociétaux et économiques auxquels la recherche doit répondre, avant de proposer des orientations qui sont ici au nombre de trois : « repousser les frontières de la connaissance »; " renforcer par l'innovation l'attractivité et la compétitivité de nos territoires »; « rapprocher recherche, innovation et société ». Les enjeux et les orientations sont déclinés en un certain nombre de défis - « défis sociétaux et économiques », d'une part, «défis de connaissances pluridisciplinaires », d'autre part -, dont chacun fait l'objet d'une fiche.

L'analyse de ces textes peut se faire à plusieurs niveaux, selon que l'on cherche à y lire les conceptions de la recherche sous-tendues par ceux-ci, la vision de l'organisation institutionnelle qu'ils proposent, ou que l'on veuille commenter le processus qui a permis l'élaboration de la SNRI. Nous ne reviendrons ici ni sur les modes de consultation et leurs mérites ou limites respectifs, ni sur les choix du gouvernement en matière d'évolution $\mathrm{du}$ fonctionnement du dispositif national de recherche et d'enseignement supérieur, choix qui imprègnent fortement ces textes mais sont bien connus et ont déjà été abondamment discutés. Au-delà de propositions procédurales ou organisationnelles qui tendent à brouiller la vision et le projet sous-jacents, ce qui peut nuire tout à la fois à l'appropriation de cette stratégie par les chercheurs et à sa résonance dans l'ensemble de la société, on peut y identifier des points de vue ou des questions renvoyant à notre projet éditorial. Nous nous contenterons donc de souligner en quoi nos préoccupations trouvent une place dans la Stratégie nationale de recherche et d'innovation.

L'interprétation de ces rapports sectoriels n'est pas simple. Tout d'abord, ces documents ne mettent en avant que des inflexions à la politique scientifique actuelle et ne font pas l'inventaire des acquis. Dès lors, une lecture un peu rapide peut donner l'impression que des pans entiers de l'activité scientifique sont complètement occultés, en particulier la recherche dite fondamentale, quelle que soit la discipline de base (sciences humaines et sociales, sciences physiques pour l'ingénieur, recherche spatiale...). Ensuite, les orientations thématiques affichées ne sont assorties d'aucune évaluation des moyens nécessaires et on ne peut donc préjuger du poids qui leur sera donné in fine : s'agit-il de jouer à la marge en impulsant quelques pistes nouvelles? S'agit-il d'infléchir en profondeur les orientations de l'activité scientifique? En d'autres termes, a-t-on affaire à une ambition scientifique inédite?

La SNRI suggère néanmoins un message assez clair. Parmi les sept défis sociétaux et économiques identifiés, le lecteur ne peut manquer d'être frappé par le poids donné aux thèmes abordés lors du Grenelle de l'environnement. Certes, la "mondialité » ainsi que la "compétitivité et l'attractivité de la France » sont identifiées, mais elles doivent être mises en regard de cinq enjeux associés au moins pour partie à ce Grenelle : "Alimentation et énergie durable »; « Ressources naturelles »; «Santé »; «Risques, aléas et sécurité des personnes, des biens et 
des communications »; "Qualité de vie des citoyens ». Il faudrait se garder d'en déduire que la SNRI est axée uniquement sur des questions d'environnement et de développement durable. Sans surprise, «l'énergie durable » ne se conçoit pas sans le nucléaire et met plus l'accent sur les technologies de production que sur les réductions de consommation, bâtiment excepté. La «qualité de la vie des citoyens » fait la part belle aux questions de transport et de mobilité, à côté de quelques considérations limitées à la ville durable. La sécurité traite très peu de risques naturels... Mais ces préoccupations sont incontestablement présentes, même si c'est avec toutes les ambiguïtés déjà dénoncées par le monde associatif lors du Grenelle de l'environnement.

De tels défis sont au cœur des principales thématiques de notre revue et les points de convergence avec les approches que nous défendons sont évidents. Ainsi, dans les fiches associées aux trois orientations, on insiste particulièrement sur l'interdisciplinarité et ses conséquences (notamment en termes d'évaluation) et sur le développement du dialogue entre sciences et société. Sur les cinq défis de connaissance ( Sciences du vivant», "Sciences de l'environnement », «Sciences et technologies innovantes autour de la matière et des matériaux », "Sciences numériques, calcul intensif et mathématiques » et «Sciences humaines et sociales face aux changements globaux»), quatre soulignent les besoins d'interdisciplinarité, en particulier les «sciences de l'environnement » et les «sciences humaines et sociales face au changement globaux». On peut se demander si cet affichage de l'interdisciplinarité n'est pas là que pour accompagner une volonté d'orienter la recherche sur des questions plus finalisées et pour rapprocher recherche et innovation. Ces deux dimensions sont en effet récurrentes tout au long des divers documents et rejoignent les positions connues du gouvernement sur le rapport public-privé, l'ancrage de la recherche dans le tissu industriel... Cependant, on ne peut ignorer le lien que proposent ces textes entre l'interdisciplinarité et la production de connaissances ou l'analyse des systèmes complexes, ce qui traduit une réelle volonté d'éviter que l'interdisciplinarité soit limitée à une instrumentalisation réciproque entre disciplines.

Enfin, le dernier point qui nous interpelle est l'analyse qui est faite des rapports recherche-société. La volonté de rapprocher recherche et innovation implique bien évidemment des contacts étroits avec les « utilisateurs » des produits de la recherche. Mais la SNRI ne se limite pas à une vision purement utilitariste de ces rapports et d'autres aspects plus conformes aux débats dont nous tentons de rendre compte apparaissent au fil des différents documents.

Claude Millier, Daniel Terrasson 